\title{
PERFIL DE COMPOSIÇÃO CORPORAL EM ATLETAS DE BASE DAS MODALIDADES DE FUTSAL E FUTEBOL DE CAMPO
}

\section{Daniel Rogério Petreça}

Núcleo de Pesquisa em Saúde Coletiva/NUPESC, Universidade do Contestado (UnC), Campus Mafra (SC) Brasil.

E-mail: profdaniel@unc.br

\section{Henrique Alexandre Ruske Neto}

Curso de Educação Física, Universidade do Contestado (UnC), Campus Mafra (SC), Brasil.
RESUMO: O objetivo deste estudo foi analisar o perfil de composição corporal em atletas de base das modalidades de "futsal" e "futebol de campo". A amostra foi composta por 244 atletas de base de 8 a 17 anos do sexo masculino. Foram realizadas medidas antropométricas de massa corporal, estatura, envergadura, dobras cutâneas de tríceps e da panturrilha-medial. Para verificar a composição corporal foram calculados o índice de massa corporal e o percentual de gordura classificando-os em protocolos de acordo com a idade. Para análise dos dados utilizou-se de estatística descritiva e inferencial. Alguns atletas praticavam as duas modalidades; para tanto, o grupo foi dividido em praticantes de futsal, futebol de campo e ambos. Foram verificadas diferenças estatísticas significativas entre os grupos na massa corporal $(p<0,0001)$, estatura $(p<0,001)$, índice de massa corporal $(p=$ $0,002)$ e envergadura $(p=0,04)$, onde o grupo que pratica ambas as modalidades obteve maiores valores. Grande parte da amostra tem o índice de massa corporal na normalidade $(77,5 \%)$, seguido de sobrepeso $(16,4 \%)$. Praticantes de ambas as modalidade obtiveram a maior prevalência de envergadura acima da média $(46,8 \%)$. No percentual de gordura a grande parte $(62,3 \%)$ obteve valores na normalidade. Os resultados demonstram uma boa composição corporal dos atletas de base. Os valores servem de parâmetro para o planejamento dos treinamentos e também de alerta em situações de saúde para aqueles que estão acima ou abaixo dos níveis aceitáveis.

PALAVRAS-CHAVE: Atletas; Composição Corporal; Crianças; Futebol.

\section{BODY COMPOSITION OF FUTSAL AND SOCCER ATHLETES}

ABSTRACT: Body composition of futsal and soccer athletes is analyzed. Sample comprised 244 male athletes, aged $8-17$ years. Anthropometric measurements for body mass, height, curve, triceps skinfold thickness and mid-calf were undertaken. Body mass index and fat percentage were calculated for body composition and classified in protocol according to age. Descriptive and inferential statistics were used for data analysis. Since several athletes practiced the two modalities, the group was divided into futsal players, soccer players and both. Different statistical differences were reported between the groups with regard to body mass $(p<0.0001)$, height $(p<0.001)$, body mass index $(p=$ $0.002)$ and curve $(p=0.04)$ in which the group which practiced the two modalities had the highest rates. Most featured normal body mass index (77.5\%), followed by overweight (16.4\%). Athletes who practiced both had the highest prevalence curve rates, above average (46.8\%). In the case of fat percentage, most athletes (62.3\%) had normal rates. Results demonstrated good body composition in the athletes. Rates may be the parameters for planning treatments and a warning in health situations for those who are below or above acceptable levels. 
KEY WORDS: Athletes; Body Composition; Children; Soccer.

\section{INTRODUÇÃO}

A obesidade na infância é um dos problemas globais mais graves do século XXI e vem aumentando em um ritmo alarmante, sendo que, mundialmente, cerca de 42 milhões de crianças com idade inferior a cinco anos estão com excesso de peso, afetando principalmente vários países de baixa e média renda em âmbitos urbanos (ORGANIZAÇÃO MUNDIAL DA SAÚDE, 2015).

Existem vários fatores que podem influenciar obesidade, como o sedentarismo, a má alimentação e fatores genéticos (VITOLO, 2008; CATANEO; CARVALHO; GALINDO, 2005), podendo acarretar a ocorrência de doenças como a hipertensão arterial, a doença cardíaca, a doença renal, diabetes mellitus tipo II (COSTA; FISBERG, 2005), a elevação dos triglicerídeos e colesterol, alterações ortopédicas, dermatológicas e respiratórias (SOARES; PETROSKI, 2003).

A obesidade e a inatividade física na infância e na adolescência podem se relacionar, uma vez que a falta de um nível de atividade física aceitável tende a elevar a gordura corporal (DELGADO; TERCEDOR, 2002). A prática regular de atividade física nesta fase de vida pode influenciar a chance de adultos mais ativos e menos sedentários (LAZZOLI, 2007).

Uma maneira de influenciar a prática de atividade física em crianças e adolescentes pode ser por meio da prática esportiva, podendo trazer benefícios duradouros na vida adulta, como bem-estar físico e mental, também evitando doenças e agravos não transmissíveis (VOSER; DE VARGAS, 2007).

A gama de opções nas práticas esportivas é imensa, dentre elas o "futsal" e o "futebol de campo" são modalidades altamente populares e praticadas no Brasil (BRASIL, 2015; DAÓLIO, 2003; BELLO 1998).

O "futebol de campo" e o "futsal" são esportes que possuem mudanças de intensidade e atividades durante a partida, variando com estilo de jogo de cada atleta (MARTIN, 2002). Para realizar sua prática, diversas valências físicas são exigidas, dentre elas estão a potência aeróbia, a potência anaeróbia, a força, a flexibilidade, a agilidade, a velocidade e também a composição corporal (HOFF, 2005; STOLEN et al., 2005; REILLY et al., 2000).

Os índices aceitáveis de composição corporal são importantes para o nível de aptidão física de atletas em qualquer categoria de treinamento e em qualquer modalidade esportiva (PRADO et al., 2006).

Portanto, levando em consideração a prevalência aumentada de obesidade na infância e adolescência e a participação efetiva de crianças e adolescentes nas modalidades de "futsal" e "futebol de campo", além da importância de traçar níveis de distúrbios de composição corporal e verificar se os projetos de práticas esportivas têm atingido praticantes de todas as características corporais ou apenas os mais aptos, esse estudo tem como objetivo analisar o perfil de composição corporal de atletas de base das modalidades de "futsal" e "futebol de campo".

\section{METODOLOGIA}

\subsection{TIPO DO ESTUDO}

O presente estudo é do tipo descritivo, observacional (survey), que visa determinar informações sobre práticas ou opiniões atuais de uma população específica. O seu valor está baseado na premissa de que os problemas podem ser resolvidos e as práticas melhoradas por meio da observação, da análise e descrição objetivas e completas (THOMAS; NELSON, 2002).

\subsection{POPULAÇÃO/AMOSTRA}

Diversos setores comunitários e privados no município de Mafra, no Estado de Santa Catarina, desenvolvem atividades de iniciação esportiva nas modalidades de futebol de campo e futsal duas vezes por semana com sessões de 90 minutos; para tanto, a população deste estudo foi formada pelos integrantes desses projetos que contabilizam cerca de 500 participantes.

Para a composição da amostra foram utilizados como critérios: autorização dos pais/responsáveis por 
meio da assinatura e entrega do Assentimento Livre e Esclarecido, realização da coleta da bateria de testes de forma voluntária. Foram considerados excluídos do estudo os atletas de base que não conseguiram realizar algum dos testes e/ou praticam outra modalidade além do "futebol de campo" e do "futsal". Para tanto, a amostra desse estudo são 244 atletas de base do sexo masculino.

\subsection{INSTRUMENTOS}

Para verificar questões relacionadas às características dos atletas de base, foi realizado um questionário contendo nome completo, data de nascimento, tempo de prática e participação em outras modalidades esportivas.

Foram realizadas medidas antropométricas de massa corporal, estatura e envergadura seguindo o protocolo da Bateria de Testes do Projeto Esporte Brasil (PROESP) (PEB, 2012) e as medidas de dobras cutâneas de tríceps e panturrilha medial de acordo com o procedimento adotado pelo The International Society for the Advancement of Kinanthropometry (ISAK ${ }^{\circledR}$ ) (MARFELL-JONES et al., 2006).

A seguir são apresentados os procedimentos de mensuração das variáveis antropométricas.

Massa Corporal (MC): para a mensuração do peso foi utilizada uma balança digital com capacidade de $180 \mathrm{~kg}$ e precisão de até 500 gramas. Foi realizada calibragem prévia a cada 10 medições. Os atletas foram medidos com trajes de treino e descalços, mantendo-se em pé com os cotovelos (braços) estendidos e juntos ao corpo. A medida foi anotada em quilogramas com a utilização de uma casa decimal.

Estatura (EST): para mensuração da estatura foi utilizada uma trena métrica de 1 metro de comprimento com precisão de até $2 \mathrm{~mm}$, fixando-a verticalmente à parede a 1 metro do solo e estendendo de baixo para cima. O atleta foi situado em pé, de costas para a parede, com as pernas e braços paralelos ao corpo, o mesmo sob o plano de Frankfurt. No final, acrescentando 1 metro (distância do solo à trena) ao resultado medido na trena métrica. A medida da estatura foi anotada em centímetros com uma casa decimal.

Envergadura (ENV): para a obtenção da medida de envergadura foi utilizada uma trena métrica com precisão de $2 \mathrm{~mm}$ anexada à parede a uma medida de 1,50 m de altura paralela ao solo, com os atletas em pé, de frente para a parede, com os braços em abdução em 90 graus o máximo possível. A medida foi registrada em centímetros do início do ponto fixo demarcado ao final da fita.

Dobras cutâneas: para a mensuração das dobras cutâneas, foi utilizado um adipômetro Innovare da marca Cescorf ${ }^{\circledR}$ resolução de $0,1 \mathrm{~mm}$, em dois locais (tríceps - DCTR e panturrilha medial - DCPM); foram realizadas duas medidas de cada dobra cutânea com o compasso em cada avaliado, registrando a média. Também foi utilizado um lápis dermográfico para marcar os pontos de pinçamento das dobras. As medidas foram realizadas do lado direito do corpo.

Para verificar a composição corporal foram utilizados o Índice de Massa Corporal - o IMC foi determinado através do cálculo da razão entre a medida de massa corporal em quilogramas pela estatura em metros elevada ao quadrado (IMC = Massa $(\mathrm{kg}) /$ estatura $\left.(\mathrm{m})^{2}\right)$, classificando conforme a tabela adaptada por Conde e Monteiro (2006). A medida é anotada com uma casa decimal.

Percentual de Gordura: para os resultados de percentual de gordura foi utilizada a equação $\% \mathrm{G}=$ $0,735(\Sigma \mathrm{TM}+\mathrm{PM})+1,0$, de Slaughter et al. (1988). A classificação do percentual de gordura foi adequada à tabela de Lohmann (1987).

\subsection{PROCEDIMENTO DE COLETA DE DADOS}

A coleta de dados iniciou após a aprovação no Comitê de Ética em Pesquisa sob protocolo $\mathrm{n}^{\mathrm{o}}$ 45207015.3.0000.0117, da Fundação Universidade do Contestado. Todos os indivíduos entregaram o Termo de Assentimento Livre e Esclarecido assinado pelos pais/ responsáveis.

A coleta de dados aconteceu na primeira semana de dezembro de 2014, nos locais e horários das sessões de treinamento (ginásio e campo de futebol) sendo disposto um local apropriado e isolado. Os atletas de base estavam vestidos com calção e camiseta.

Inicialmente foi realizada a demarcação de 
pontos anatômicos (acromiale, radiale, mid-acromialeradiale, maior perímetro da panturrilha e panturrilha medial). A sequência das medidas antropométricas foi massa corporal, estatura, envergadura e dobras cutâneas de tríceps e panturrilha medial.

As medidas foram realizadas por um único avaliador (estudante do curso de Educação Física), o qual passou por treinamento prévio (12 horas) por 01 instrutor com Certificação Internacional em Antropometria da ISAK ${ }^{\circledR}$ Level 3 .

\subsection{ANÁLISE ESTATÍSTICA}

Os dados foram organizados em planilhas do programa Microsoft Excel 2010 ${ }^{\circledR}$ sendo em seguida transportados para o programa estatístico IBM SPSS ${ }^{\circledR}$ 20.0. Para tanto, foram realizadas análises descritivas e inferenciais. O teste de KolmogorovSmirnov foi utilizado para verificar normalidade. Para verificar diferença de médias entre as variáveis em relação às modalidades "futsal", "futebol" e "futsal e futebol" foi utilizada análise de variância ANOVA para as variáveis paramétricas e Kruskal-Wallis para as não paramétricas.

\section{RESULTADOS}

Participaram deste estudo atletas de base sendo verificado que $37,7 \%$ praticam a modalidade de "futsal", $36,9 \%$ praticam a modalidade de "futebol de campo", e $25,4 \%$ praticam "ambas" as modalidades. Em relação à idade obteve-se média de 12,3 (DP = 2,2) anos obtendo valor mínimo de 8 e máximo de 17 anos, não ocorrendo diferença significativa entre os grupos de praticantes.

Ao dividi-los em faixas etárias, $41,4 \%$ estão entre 8 a 11 anos, 37,3\% estão entre 12 a 14 anos e 21,3\% entre 15 a 17 anos. Ao verificar o tempo de prática nas modalidades, 43,6\% têm até um ano, 26,7\% entre 1 a 2 anos, e 29,6\% acima de 3 anos.

A Tabela 1 apresenta os dados descritos das variáveis antropométricas identificando a análise de variância entre os praticantes de "futsal", "futebol de campo" e "ambas" além dos valores gerais da amostra.

Para tanto, obteve-se diferenças significativas entre as variáveis antropométricas na MC $(p<0,001)$, EST $(p<0,001)$, IMC $(p=0,002)$ e ENV $(p=0,03)$, sendo o grupo que pratica "ambas" as modalidades com maiores valores: $52,9 \mathrm{~kg}, 162,4 \mathrm{~cm}, 19,7 \mathrm{~kg} / \mathrm{m}^{2}$ e 155,6 $\mathrm{cm}$, respectivamente. Os testes de DCTR, DCPM e PERC não apresentaram diferenças entre as modalidades.

Tabela 1. Apresentação descritiva das variáveis e índices antropométricos dos atletas de base da modalidade de futsal e campo

\begin{tabular}{lcccccccc|c}
\hline & \multicolumn{2}{c}{$\begin{array}{c}\text { Futsal } \\
\mathbf{n = 9 2}\end{array}$} & \multicolumn{2}{c}{$\begin{array}{c}\text { Futebol } \\
\mathbf{n = 9 0}\end{array}$} & \multicolumn{2}{c}{$\begin{array}{c}\text { Ambas } \\
\mathbf{n = 6 2}\end{array}$} & \multicolumn{2}{c}{$\begin{array}{c}\text { Geral } \\
\mathbf{n = 2 4 4}\end{array}$} & \multirow{2}{*}{$\boldsymbol{p}$-valor } \\
\cline { 2 - 8 } & Média & DP & Média & DP & Média & DP & Média & DP & \\
\cline { 2 - 8 } MC & 44,9 & 13,7 & 42,8 & 15,4 & 52,9 & 13,4 & 46,2 & 14,8 & $<0,001^{*}$ \\
EST & 153,6 & 13,9 & 150,8 & 17,7 & 162,4 & 13,4 & 154,8 & 15,9 & $<0,001^{*}$ \\
IMC & 18,6 & 3,4 & 18,2 & 3,3 & 19,7 & 3,0 & 18,7 & 3,3 & $0,002^{*}$ \\
ENV & 146,8 & 16,3 & 149,5 & 19,3 & 155,6 & 22,0 & 150,1 & 19,2 & $0,03^{*}$ \\
DCTR & 10,7 & 4,8 & 10,0 & 4,3 & 10,3 & 5,2 & 10,3 & 4,7 & 0,6 \\
DCPM & 11,2 & 5,2 & 10,4 & 5,0 & 10,2 & 5,3 & 10,7 & 5,2 & 0,2 \\
PERC & 17,1 & 7,2 & 16,0 & 6,6 & 16,1 & 7,5 & 16,4 & 7,0 & 0,4 \\
\hline
\end{tabular}

Legenda: DP: desvio padrão; MC: Massa Corporal (kg); EST: Estatura (cm); IMC: Índice de Massa Corporal (kg/m²); ENV: Envergadura; DCTR: Dobra Cutânea Tríceps (mm); DCPM: Dobra Cutânea Panturrilha Medial (mm); PERC: Percentual de Gordura (\%); * diferença estatística significativa.

A Figura 1 apresenta o IMC de acordo com os pontos de corte divididos em "baixo peso", "normal", "sobrepeso" e "obesidade". Observa-se que 80,4\% dos praticantes de "futsal" foram classificados como "normal", em contraponto obtiveram a maior prevalência na obesidade (6,5\%). Os praticantes de "ambas" as modalidades obtiveram a maior prevalência no sobrepeso $\operatorname{com} 24,2 \%$. 


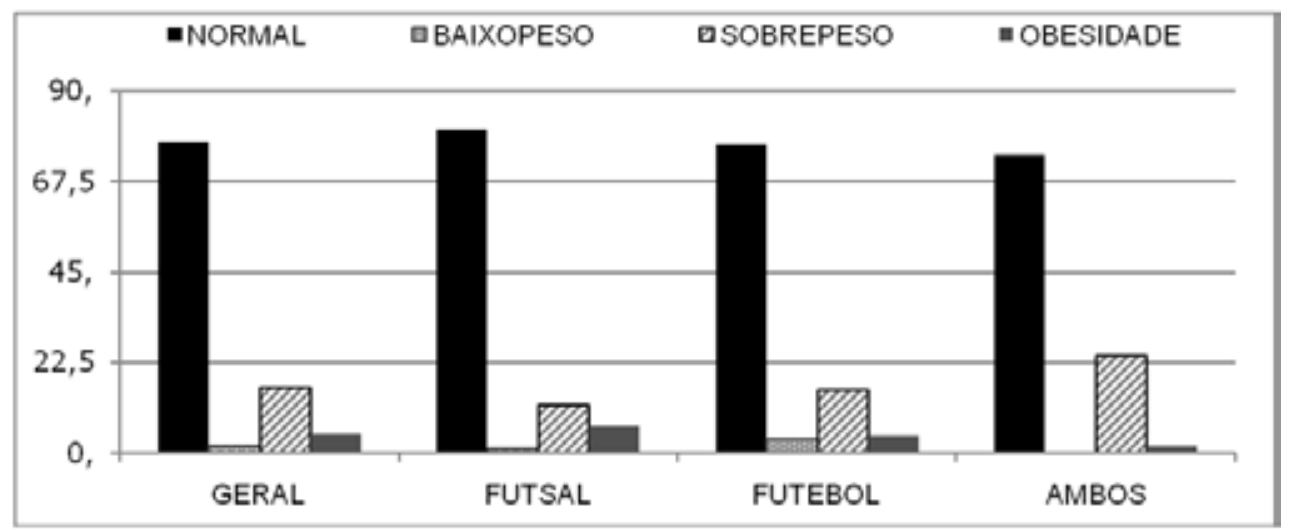

Figura 1. Análise de frequência em relação aos pontos de corte estabelecidos para o IMC dos atletas de base de futsal, futebol de campo e praticante de ambas

A Figura 2 apresenta dados de frequência da para os praticantes de "futebol". Os praticantes de envergadura. Os praticantes de "futsal" apresentaram "ambas" as modalidade obtiveram os maiores índices de 31,5\% "abaixo da média" tendo uma diferença de 7,4\% prevalência "acima da média" com 46,8\%.

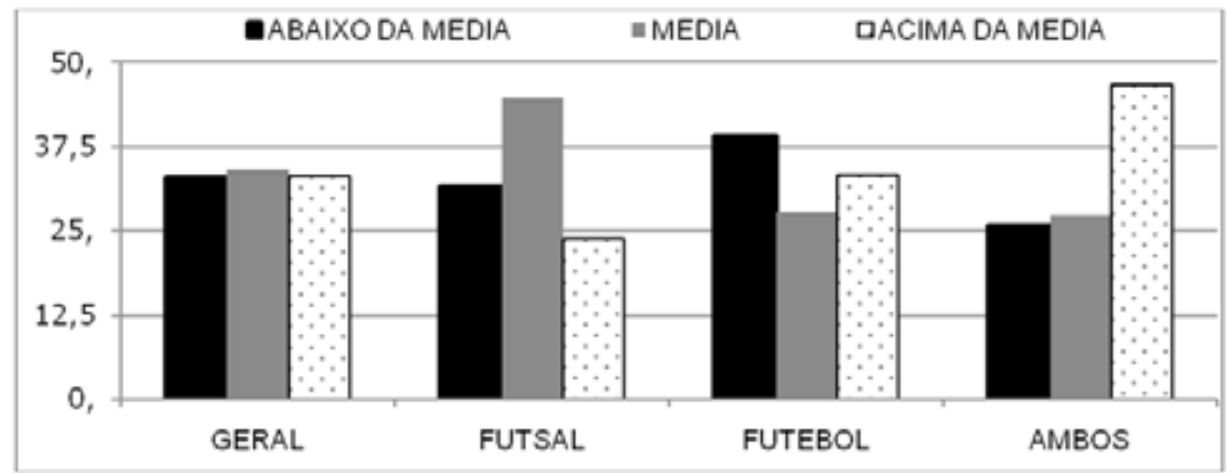

Figura 2. Análise de frequência da envergadura dos atletas de base de futsal, futebol de campo e praticante de ambas

A Figura 3 apresenta dados de frequência do Em contraponto, foram observados atletas classificados percentual de gordura. Grande parte da amostra possui como "moderadamente alto" (12,3\%), "alto" (5,7\%) e o percentual de gordura em níveis "normais" (66,1\%). "excessivamente alto" (4,9\%).

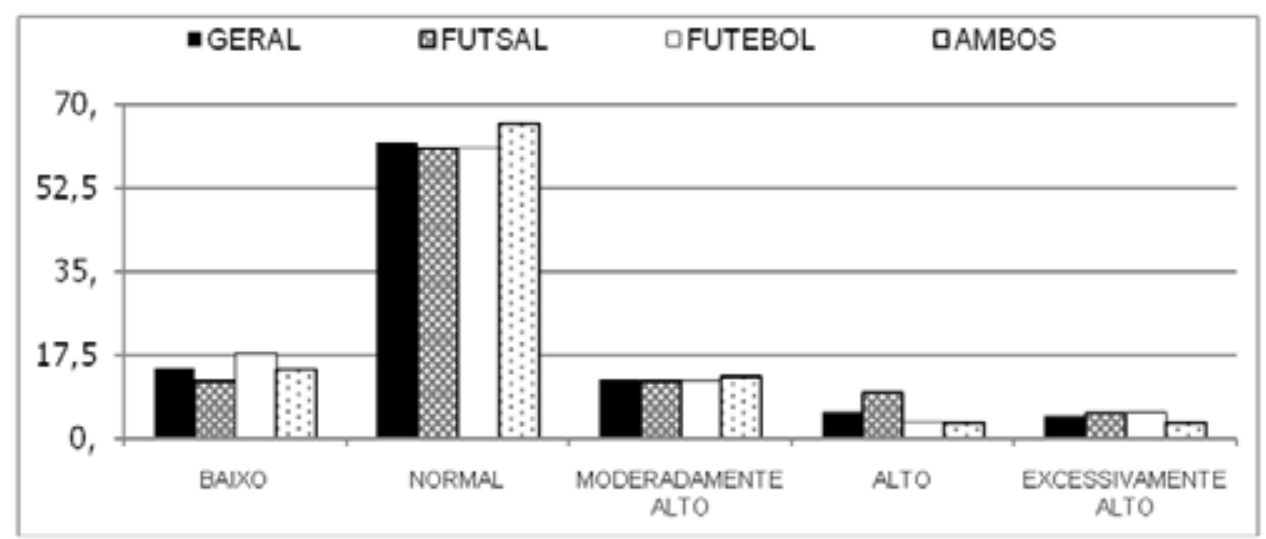

Figura 3. Análise de frequência do percentual de gordura dos atletas de base de futsal, futebol de campo e praticante de ambas 


\section{DISCUSSÃO}

Este estudo buscou analisar o perfil de composição corporal de atletas de base das modalidades de "futsal" e "futebol de campo", levando em consideração participantes simultâneos das modalidades, por meio de medidas antropométricas e avaliações de parâmetros como o Índice de Massa Corporal, a envergadura e o percentual de gordura.

Algumas limitações foram observadas, como a não mensuração dos hábitos alimentares, nem a possível participação efetiva dos atletas de base enquanto prática escolar nas aulas de Educação Física. Além disso, as análises de prevalência foram realizadas pela idade cronológica, pois não foi possível realizar avaliação maturacional da amostra.

Enfim, o esporte é uma oportunidade da criança e do adolescente estar fisicamente ativo, sendo recomendado pelo Center for Disease Control and Prevention (CDC, 2011) dos Estados Unidos, que práticas mais ativas, como o futebol, sejam incorporadas tanto no currículo escolar, extracurricular, como nos momentos de lazer.

O futsal e o futebol de campo são esportes que possuem várias exigências físicas e motoras, e segundo Prado et al. (2006), devido à sua duração, pode promover um gasto calórico alto de seus praticantes, tanto em dias de jogos, quanto em treinamentos, o que pode contribuir, segundo Guinhouya (2012), para a prevenção da obesidade, pois segue associação inversa entre nível de atividade física e seu desenvolvimento.

Em relação ao Índice de Massa Corporal utilizado nesse estudo, pois, segundo a World Health Organization (1995), é um método fácil e de baixo custo de grande importância para avaliação do estado nutricional de crianças e adolescentes, foi possível verificar padrões de normalidade em $77,5 \%$ da amostra.

Rodrigues et al. (2010), em um estudo realizado em uma escola do Rio Grande do Sul e uma escola no Paraná, com 100 jovens de 13 a 15 anos de idade, observaram nos atletas de futsal valores de $84,2 \%$ deles na classificação normal, índice próximo dos 80,4\% obtidos nesse estudo aos atletas da mesma modalidade.

Objetivando conhecer níveis de aptidão física, saúde e desempenho motor de 30 praticantes de futebol ou futsal entre 14 e 15 anos no município de Caxias do
Sul (RS), Generosi et al. (2007) verificaram que os 30 atletas de futsal obtiveram média de IMC de $20,6 \mathrm{~kg} / \mathrm{m}^{2}$, superior a este estudo, que obteve 18,7 e $18,6 \mathrm{~kg} / \mathrm{m}^{2}$, nos atletas de futsal e geral, respectivamente, o que pode ser explicado pela diferença de cerca de dois anos na média de idade (12,2 anos).

As maiores prevalências para sobrepeso foram observadas nos praticantes de ambas as modalidades $(24,2 \%)$, maior que a média nacional de $21,7 \%$ e na obesidade no futsal (6,5\%), maior que a média nacional de 5,9\% (IBGE, 2009).

A obesidade pode ser associada a diversas patologias, entretanto, segundo Wake et al. (2013), estar abaixo do peso é relacionado substancialmente com maior morbidade do que a obesidade em crianças. Os atletas praticantes da modalidade de futebol obtiveram a maior prevalência em baixo-peso, com 3,3\%, sendo menor que a média nacional de 3,7\% (IBGE, 2009).

Os atletas que praticavam ambas as modalidades obtiveram os maiores valores para massa corporal, estatura e IMC, o que pode ser explicado que, apesar de não haver diferença significativa entre as idades e os grupos, eram os indivíduos mais velhos.

Além de situações relacionadas à saúde, o conhecimento do perfil de composição corporal e de seus respectivos comportamentos e respostas ao processo de treinamento, segundo Mortatti et al. (2013), é fundamental para o contexto da especialização esportiva e de grande relevância para um bom rendimento (MORAES; HERDY; SANTOS, 2009). Como o desempenho desportivo evolui, as preocupações com os fatores envolvidos na sua melhoria aumentam, de modo que o monitoramento de variáveis de composição corporal para alcançar melhores resultados se torna mais importante (MALINA et al., 2004).

Neste estudo, foram mensurados a fim de desempenho, a envergadura e o percentual de gordura. Em relação à envergadura, ela é influenciada pela idade do indivíduo (OELKE; KROEFF, 2006), sendo demonstrado que, segundo Kirk (2016), um atleta que tem uma envergadura maior que sua estatura tem vantagens, sendo pré-requisito para o sucesso em determinadas modalidades esportivas, caso do basquetebol, handebol e voleibol, e que, de acordo com o estudo de Silva et 
al. (2013), foi possível verificar que os praticantes dessa modalidade tinham significativamente maior envergadura do que aqueles que escolheram o futebol.

Nesse estudo, a envergadura foi constatada em nível médio e acima da média em 67,2\% da amostra, obtendo o maior nível nos atletas praticantes de ambas as modalidades, sendo $46,8 \%$ acima da média com valor de $155,6 \mathrm{~cm}$. Em comparação, estudo realizado com Grando e Martins (2012) em 25 atletas do sexo masculino da categoria sub 12 e sub 13 da modalidade de futsal, no município de Guarapuava (PR), obteve média de envergadura de $154,0 \mathrm{~cm}, 7,2 \mathrm{~cm}$ superior a este estudo.

Valores de envergadura foram observados em atletas de futsal também no estudo de De Jesus et al. (2010), com 33 jovens de 9 a 13 anos de idade no município de Curitiba (PR), apresentando valor médio de $149,0 \mathrm{~cm}$, para a categoria sub $11 \mathrm{de} 152,0 \mathrm{~cm}$ e na categoria sub 13 o valor de $152,07 \mathrm{~cm}$.

Em relação ao percentual de gordura, segundo Prado et al. (2006) um maior percentual pode diminuir substancialmente o desempenho esportivo. Apesar disso, foi levado em consideração neste estudo a dificuldade de se analisar alguns componentes nas faixas etárias estabelecidas e que, segundo Silva et al. (2004), dificulta a interpretação do impacto dos esportes em virtude dos diferentes estágios puberais além do número de atividades físico-desportivas potenciais e suas variadas formas.

Apesar disso, este estudo demonstrou percentuais de gordura que variam em média de $16,4 \%$. Valores menores foram encontrados no estudo realizado por Mantovani et al. (2008) com 37 atletas de base de futebol em São Paulo, onde 18 atletas da categoria sub-15 apresentaram 8,2\% de gordura e 19 atletas da categoria sub-17 apresentaram 7,9\% de gordura.

Entretanto, nossos valores foram bem menores do que os encontrados por Machado (2010), com 94 jovens do sexo masculino, 43 praticantes de futebol de campo e 51 praticantes de futsal no município de Cianorte (PR), onde o resultado para o percentual de gordura foi, em média, de 23,3\%.

Por fim, nosso estudo demonstrou que 25,4\% praticam "ambas" as modalidades, ou seja, realizam treinamentos de aproximadamente 240 minutos por semana, o que parece ser significante para uma rotina diária ativa, a qual se recomendam, segundo o American College of Sports Medicine (2013), atividades aeróbias de moderadas à vigorosas por mais de 60 minutos por dia.

Indivíduos nessa faixa etária, segundo Silva (2013) em uma revisão sistemática sobre a inatividade física na adolescência, demonstram alto dispêndio de tempo desse público em atividades de comportamento sedentário como assistir televisão, computador e videogames pelo tempo superior aos recomendados como ideais à saúde.

\section{CONCLUSÃO}

Nosso estudo verificou diferenças estatísticas significativas entre os grupos na massa corporal, estatura, índice de massa corporal e envergadura. Além disso, grande parte da amostra apresentou o índice de massa corporal na normalidade. Praticantes de ambas as modalidades obtiveram a maior prevalência de envergadura acima da média e no percentual de gordura a grande parte obteve valores na normalidade.

Os valores obtidos se tornam importantes para profissionais de Educação Física, treinadores em escolas de esporte, a fim de analisar situações futuras e adversas tanto relacionadas à saúde quanto ao desempenho esportivo servindo de parâmetro para o planejamento dos treinamentos além da possível comparação com atletas de outras categorias, modalidades e regiões.

\section{REFERÊNCIAS}

AMERICAN COLLEGE OF SPORTS MEDICINE (ACSM) ACSM's guidelines for exercise testing and prescription. [s.1.]: Lippincott Williams \& Wilkins, 2013.

GUINHOUYA, B. C. Physical activity in the prevention of childhood obesity. Paediatr Perinat Epidemiol, v. 26, p. 438-447, 2012.

BELLO, J. R. N. A ciência do esporte aplicada ao futsal. Rio de Janeiro: Sprint, 1998. 
BRASIL. Diagnóstico Nacional do Esporte. Ministério do Esporte do Brasil. Disponível em: <http://www. esporte.gov.br/diesporte/index.html >. Acesso em: dez. 2015.

CataneO, C.; CARVAlHO, A. M. P.; Galindo, E. M. C. Obesidade e Aspectos i Psicológicos: Maturidade Emocional, Auto-conceito, Locus de Controle e Ansiedade.

Psicol. Reflex. Crit., v. 18, n. 1, p.39-46, 2005.

CENTERS FOR DISEASE CONTRAMEOL AND PREVENTION (CDC) School health guidelines to promote healthy eating and physical activity. MMWR. Recommendations and reports: Morbidity and mortality weekly report. Recommendations and reports/Centers for Disease Control, v. 60, n. R-5, p. 1, 2011.

COSTA, R. F.; FISBERG, M. Atividade física e obesidade. In: ATIVIDADE física adaptada. Barueri: Manole, 2005.

DAÓLIO, J. Cultura: educação física e futebol. 2. ed. Campinas: Unicamp, 2003.

DE JESUS, K.; DE JESUS, K.; DE JESUS, H. Caracterização cineantropométrica dos jogadores de futsal paranaense das categorias sub 11 e sub 13. Lecturas Educación Física y Deportes (Buenos Aires), v. 15, p. 1-1, 2010.

DELGADO, M.; TERCEDOR, P. Estrategias de intervecion en educacion para la salud desde a educacion física. Barcelona: INDE Publicaciones, 2002.

GENEROSI, R. A.; BARONI, B. M.; GROSSELLI, D.; MARCHI, T.; LEAL JUNIOR, E. C. P.; NAVARRO, F. Níveis de aptidão física relacionada à saúde e ao desempenho motor de XØadolescentes praticantes de futebol e futsal. Rev. Bras. Futsal e Futebol, v. 1, n. 3, p. 251-258, set./ dez. 2009.

GRANDO, D.; LACERDA MARTINS, J. C. Análise dos níveis de aptidão física de meninos praticantes de futsal na categoria sub 12-13 da cidade de Guarapuava - PR. Revista Voos, v. 3, n. 2, 2012.

HOFF, J. et al. Soccer specific aerobic endurance training. Br J Sports Med, Surrey. v. 36, p. 218-221, 2005.
IBGE. Pesquisa de Orçamentos Familiares 20082009. Antropometria e estado nutricional de crianças, adolescentes e adultos no Brasil. Disponível em: $<$ http://www.ibge.gov.br/home/estatistica/populacao/ condicaodevida/pof/2008_2009_encaa/comentario. pdf $>2009$. Acesso em: 14 jun. 2015.

KIRK, C. Does stature or wingspan length have a positive effect on competitor rankings or attainment of world title bouts in international and elite mixed martial arts? PeerJ Preprints, v. 1, n. e., 1892, 2016.

LAZZOLI, J. K. Título de Especialista em Medicina Esportiva Sociedade Brasileira de Medicina do Esporte. Rev Bras Med Esporte, v. 13, n. 1, p. 6-8, 2007.

LOHMANN, T. G. The use of skinfold to estimate body fatness on children and youth. JOPERD, v. 58, n. 9, p. 98-103, 1987.

MACHADO, V. H. R. Características dos participantes e das atividades esportivas nas modalidades de futebol de campo e salão. 2010. Dissertação (Mestrado) - Universidade Federal do Paraná, Programa de PósGraduaçao em Educaçao Física. 2010.

MALINA, R. M.; E, J. C.; CUMMING, S. P.; RIBEIRO, B.; AROSO, J. Maturity-associated variation in the growth and functional capacities of youth football (soccer) players 1315 years. Eur. J. Appl. Physiol, v. 91, p. 555-562, 2004.

MANTOVANI, T. V. L.; RODRIGUES, G. A.; MIRANDA, J. M.; PALMEIRA, M. V.; ABAD, C. C.; WICHI, R. B. Composição corporal e limiar anaeróbio de jogadores de futebol das categorias de base. RREMEFE, v. 7, p. 25-33, 2008.

MARFELL-JONES, M. International standards for anthropometric assessment. Potchefstroom. South África: ISAK, 2006.

MARTIN, V. Futebol: lactato e amônia sanguíneos em teste de velocidade supra-máxima. 2002. Dissertação (Mestrado) - Universidade de São Paulo, Escola de Educação Física e Esporte, São Paulo, 2002.

MORAES, M. V. L.; HERDY, C. V.; SANTOS, M. P. Análise dos aspectos antropométricos em jovens atletas de alto rendimento praticantes da modalidade futebol. $\mathbf{R}$. bras. 
Ci. E Mov. v. 17, n. 2, p. 100-107, 2009.

MORTATTI, ARNALDO L. et al. El uso de la maduración somática en la identificación morfofuncional en jóvenes jugadores de fútbol. Rev Andal Med Deporte, v. 6, n. 3, p. 108-114, 2013.

OELKE, A. S.; KROEFF, M. S. Associação entre envergadura e o desempenho motor no teste de golpeio de placas. Lecturas: Educación física y deportes, n. 93, p. 31, 2006.

PRADO, W. L.; BOTERO, J. P.; GUERRA, R. L. F.; RODRIGUES, C. L.; CUVELLO, L. C.; DAMASO, A. R. Perfil antropométrico e ingestão de macronutrientes em atletas profissionais brasileiros de futebol, de acordo com suas posições. Rev Bras Med Esporte, v. 12, n. 2, p. 61-65, 2006.

PROJETO ESPORTE BRASIL. PROESP. Manual de testes e avaliação. Porto Alegre: Escola de Educação Física da UFRGS, 2012.

REILLY, T.; BANGSBO, J.; FRANKS, A. Anthropometric and physiological predispositions for elite soccer. J Sports Sci., v. 18, n. 9, p. 669-683, 2000.

RODRIGUES, D. C.; PAULA, C. V.; LIBERALI, R.; ALMEIDA, R. Comparação do perfil antropométrico de atletas e não atletas de futsal adolescentes de escolas no Rio Grande do Sul e Paraná. RBFF, v. 2, n. 4, p. 37-41, 2010.

SILVA, J. V. P. D. (In) Atividade física na adolescência: revisão sistemática. Rev. bras. ciênc. mov, v. 21, n. 3, p. 166-179, 2013.

SILVA, C. C.; GOLDBERG, T. B.; TEIXEIRA, A. S.; MARQUES, I. Does physical exercise increase or compromise children's and adolescent's linear growth? Is it a myth or truth? Rev Bras Med Esporte, v. 10, p. 520-524, 2004.

SILVA, D.; PETROSKI, E.; GAYA, A. Anthropometric and Physical Differences Among Brazilian Adolescents who Practice Different Team Court Sports, J Hum Kinet., v. 26, p. 77-86, 2013.

SLAUGHTER, M. H.; LOHMAN, T. G.; BOILEAU, R. A.;
HORSWILL, C. A.; STILLMAN, R. J.; VAN LOAN, M. D..; BEMBEN, D. A. A Skinfold equations for estimation of body fatness in children and youth. Human Biology, v. 2, n. 1, p. 7-17, 1988.

SOARES, L. D.; PETROSKI, E. L. Prevalência, Fatores Etiológicos e Tratamento da Obesidade Infantil, Rev bras cineantropom desempenho hum., , v. 5, n. 1, p. 63-74, 2003.

STOLEN, T.; CHAMARI, K.; CASTAGNA, C.; WISLOFFU. Physhiology for soccer: An uptade. Sports Med., v. 35, p. 501-36, 2005.

THOMAS, J. R.; NELSON, J. R. Métodos de pesquisa em atividade física. 3. ed. Porto Alegre: Artmed, 2002.

VITOLO, M. R. Nutrição da gestação ao envelhecimento. Rio de Janeiro: Rubio, 2008.

VOSER, R. C.; VARGAS, L. A. M. A Criança submetida precocemente no esporte: benefícios e malefícios. [s. 1.]: Futsal Brasil, 2007.

WAKE, M.; CLIFFORD, S. A.; PATTON, G. C.; WATERS, E.; WILLIAMS, J.; CANTERFORD, L.; CARLIN, J. B. Morbidity patterns among the underweight, overweight and obese between 2 and 18 years: population-based cross-sectional analyses. Int J Obes (Lond), v. 37, n. 1, p. 86-93, 2013.

WORLD HEALTH ORGANIZATION. Status: Global Strategy on Diet, Physical Activity and Health: Childhood overweight and obesity: WHO, 2015. Disponível em: $<$ http://www.who.int/dietphysicalactivity/childhood/ en/> Acesso em: 04 maio 2015.

WORLD HEALTH ORGANIZATION. Physical Status: The use and interpretation of anthropometry. Geneva, Switzerland: WHO, 1995. (WHO Technical Report Series, n. 854).

Recebido: 22 de fevereiro de 2016 Revisado: 01 de abril de 2016 Aceito: 20 de abril de 2016 